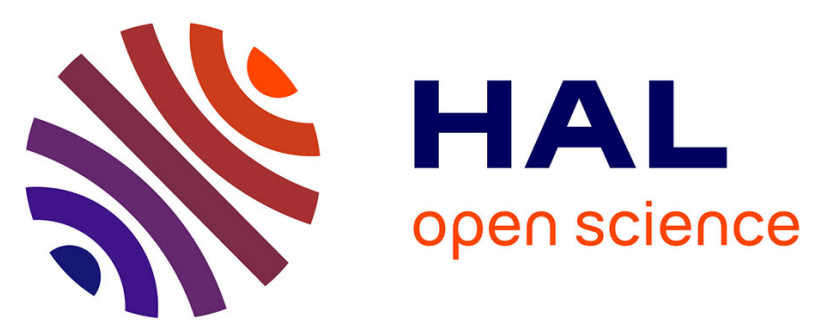

\title{
Capacitive Behavior of HF Power Transformers: Global Approach to Draw Robust Equivalent Circuits and Experimental characterization
}

\author{
Abdelhadi Besri, Hervé Chazal, Jean-Pierre Kéradec
}

\section{To cite this version:}

Abdelhadi Besri, Hervé Chazal, Jean-Pierre Kéradec. Capacitive Behavior of HF Power Transformers: Global Approach to Draw Robust Equivalent Circuits and Experimental characterization. IEEE Instrumentation \& Measurement Technology Conference, May 2009, Singapour, Singapore. hal00385355

\section{HAL Id: hal-00385355 \\ https://hal.science/hal-00385355}

Submitted on 19 May 2009

HAL is a multi-disciplinary open access archive for the deposit and dissemination of scientific research documents, whether they are published or not. The documents may come from teaching and research institutions in France or abroad, or from public or private research centers.
L'archive ouverte pluridisciplinaire $\mathbf{H A L}$, est destinée au dépôt et à la diffusion de documents scientifiques de niveau recherche, publiés ou non, émanant des établissements d'enseignement et de recherche français ou étrangers, des laboratoires publics ou privés. 


\title{
Capacitive Behavior of HF Power Transformers:
}

\section{Global Approach to Draw Robust Equivalent Circuits and Experimental characterization}

\author{
A.Besri, H.Chazal and J-P. Keradec, senior member, IEEE \\ Laboratoire de Génie Electrique de Grenoble (G2Elab), UMR 5269 INPG-UJF-CNRS \\ 38402 Saint Martin d'Hères Cedex, France \\ besri@g2elab.grenoble-inp.fr
}

\begin{abstract}
This paper addresses the representation and the characterization of the electrostatic behavior of n-windings $\mathrm{HF}$ power transformers. A global approach, mainly based on energy considerations about linear electrostatic circuits, is introduced. It leads to equivalent circuits which include minimum numbers of elements. Besides, owing to a special approximation that is often acceptable, the general circuit can be greatly simplified and so it is for the experimental identification. This is discussed. Then, knowing the topology of the needed circuits, we show how to experimentally find the values of all involved components. This is achieved by using a wide frequency range impedance analyzer according to a well defined process. An industrial 3-winding transformer is used to illustrate this identification process and, finally, curves related to the equivalent circuit are compared to experimental ones.
\end{abstract}

Keywords-HF power transformer, equivalent circuit, stray capacitances, capacitance matrix, impedance measurements.

\section{INTRODUCTION}

In order to reduce size and weight of equipments, operating frequencies of switching power supplies are getting higher and higher so, today, designers must, quite always, account for capacitive behavior of all components. Among these components, HF power transformers constitute a special case. Even if, in normal operating conditions, they behave linearly, they often have a lot of terminals so drawing an equivalent circuit to model their electrostatic behavior is not straightforward.

As it can be seen in [1], attention to electrostatic properties of HF power transformers has already been paid by numerous authors. These studies can be ranked into two complementary categories. For the first one, the target is to deduce one or several capacitances from the geometrical-physical description of the component. Second one aims at finding a general representation which accounts for the whole observable electrostatic behavior of the component. Following this last approach, a reduced set of parameters is deduced from measurements. To sum up, while the second approach teaches how to experimentally characterize the electrostatic behavior by a few parameters, the first one shows how to deduce these parameters from the description of the component.
Our team is studying the representation of wound components since the early 90's [2], [3] and [4]. Recently, we published a synthetic paper dedicated to the representation of magnetic coupling [5] and another one [6] focused on its experimental identification. In this paper, we will pay attention to capacitive effects. To represent all these effects, from the beginning we adopted a global approach which belongs to the second kind of studies.

Most of HF power transformers use ferrite core or no core at all. In these circumstances, assuming their electrical behavior is linear is a suitable and powerful hypothesis. As long as it behaves linearly, the transformer can be represented by an equivalent circuit which only includes linear impedances. Indeed, it becomes non linear only when the component goes out of its normal operation conditions so the induction approaches its saturation value. All our representations aim to model the linear behavior that can be described, at every frequency, by a set of impedances (Figure 1). Because a transformer is generally used in the frequency range in which it is inductive, we call "maximum working frequency" $\left(f_{\text {omax }}\right)$, the lower parallel resonance frequency which appears on these Bode plots.

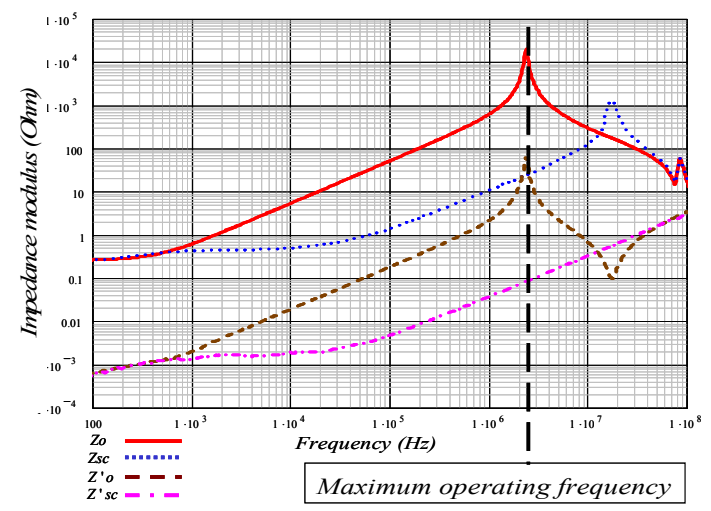

Figure 1. Open and short circuit impedance modulus measurements

A key point of our representations is that we always assume a capacitive system is connected in parallel with a magnetic system which includes losses (Figure 2). Such a splitting proved to be reliable up till one and, even, two decades beyond

This work was supported by THALES AIRBORNE

SYSTEMS - Brest, FRANCE 
$f_{\text {omax }}$ Magnetic part has already been described [5] [6] so, in this paper, we focus on capacitive coupling.

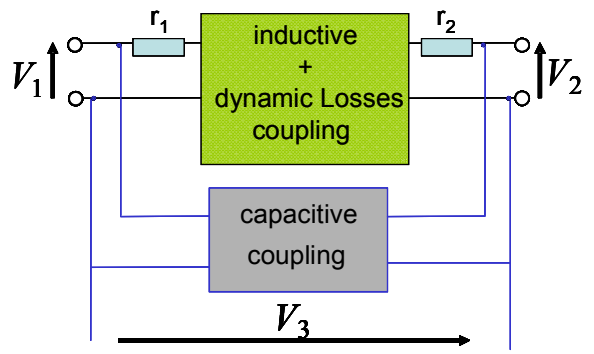

Figure 2. Splitting the equivalent circuit of a 2-winding transformer

In section II we present the physical considerations that lead to a first global model of the capacitive behavior of a transformer. We also mention experimental facts that strengthen the global approach. Section III shows how accurate measurements can lead to wrong capacitance values. A simple rule is given to choose measurements which avoid this problem. The progressive representation is introduced in section IV. This approach split the full identification in two successive steps. The first one exploits measurements acquired at easily accessible frequencies and it gives a satisfying approximation to represent the capacitive behavior. The second one leads to the most precise representation when measurements at higher frequencies are possible. Section V focuses on inequalities that capacitance matrix elements must satisfy. The way they can be verified on the final representation is also exposed. Finally, section VI shows real measurements and checks model-experiment agreement.

\section{BASIC REPRESENTATION OF THE CAPACITVE BEHAVIOR}

\section{A. Elementary considerations}

Now let us focus on linear electrostatic n-port device. Assuming it is lossless, electrical behavior of such a system is fully described by its capacitance matrix $[C](1)$ and the energy it stores $W$ is a quadratic function of all input voltages (2).

$$
[I]=s[C][V] \text { with } C_{j i}=C_{i j}
$$

Where: $\mathrm{s}$ is the Laplace variable.

$$
W=\frac{1}{2}[V]^{t}[C][V]
$$

So, two electrostatic systems having always the same energy have also the same capacitance matrix and their capacitive behaviors are identical. Our first type of representation comes from this idea. We draw an electrostatic equivalent circuit by placing a capacitor between each couple of terminals. In this way, the number of capacitor equals that of independent elements of the matrix.

\section{B. The two-winding transformer}

For a two winding transformer that has three electrostatic inputs (inter-winding voltage matters!), the circuit includes six capacitors (Figure 3) and stored energy is given by (3).

$$
\begin{aligned}
W= & \frac{1}{2}\left(C_{1}+C_{4}+C_{5}\right) V_{1}^{2}+\frac{1}{2}\left(C_{2}+C_{4}+C_{6}\right) V_{2}^{2}+\frac{1}{2}\left(C_{3}+C_{4}+C_{5}+C_{6}\right) V_{3}^{2} \\
& -C_{4} V_{1} V_{2}-\left(C_{4}+C_{5}\right) V_{1} V_{3}+\left(C_{4}+C_{6}\right) V_{2} V_{3}
\end{aligned}
$$

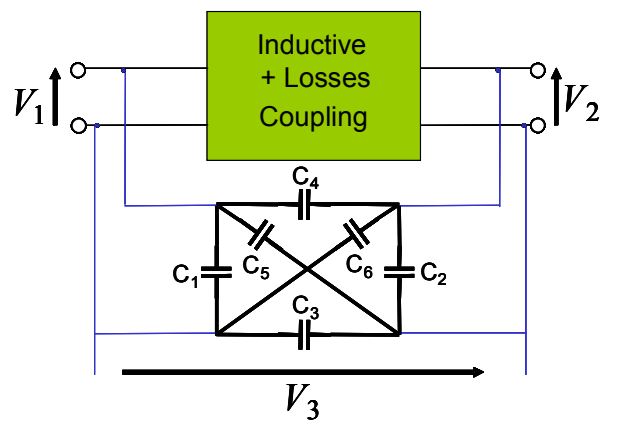

Figure 3. Elementary representation of electrostatic behavior for a two winding transformer

Identifying (2) and (3), capacitors $C_{i}$ can be expressed in terms of $C_{i j}$ and reversely. The six parameters $C_{i}$ can be deduced from impedance measurements. Obviously, interwinding capacitances can be measured directly and, if magnetic equivalent circuit has been identified first [6], parallel resonances of windings allow other values of capacitances to be deduced. That way, a sufficient number of independent equations is obtained to solve the problem. It should be noted that, at this point, some among the six capacitors can be negative. This will be discussed in depth in section $\mathrm{V}$.

\section{Experimental evidences}

To illustrate the global approach, let us consider a two winding transformer in which each winding is made of one layer (Figure. 4). Electrostatic energy is mainly stored inside the parallelepiped limited by the layers and, along both layer sides, voltage varies linearly (same voltage per turn). Laplace's electrostatic equation [2] leads to electrical field and, then, to energy. Finally, identification with (3) gives capacitor values shown in figure 4.

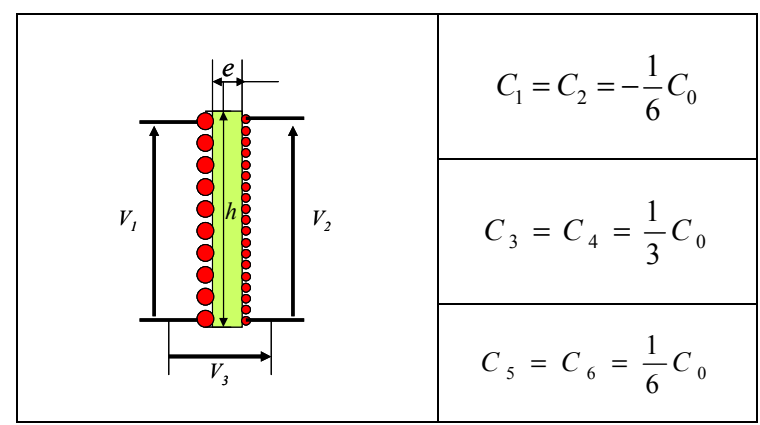

Figure 4. Transformer with two one layer windings

This shows that, for this common geometry, some capacitances are negative. Of course, directly measurable ones are not! Moreover, looking at the energy stored when first, secondary is floating and second, when $V_{3}=0$, shows a 4 ratio. This has been confirmed experimentally by looking at the first pole of the open circuit impedance: its frequency decreases in a 2.03 ratio (theoretically 2 ) when linking the low terminals of both windings $\left(V_{3}=0\right)$. This is a first success of the global approach. 
Whatever the winding number may be, adding admittance matrixes related to inductive and capacitive couplings, allows systematic equalities of resonance frequencies to be explained. For example, for a two winding transformer, the first zero of the open circuit impedance seen from one winding occurs at the same frequency as the first pole of the short circuit impedance seen from the other winding (Figure 1). These equalities are always accurately satisfied in practice; this is a second success of the global approach.

\section{WRONG SOLUTIONS AND CHOICE OF MEASUREMENTS}

\section{A. Experimental identification of an elementary device}

The simplest linear electrostatic device which involves a coupling is represented as in figure 7. If we limit externally added impedances to short circuits, six different impedances (capacitances) can be measured: three with no short-circuit, three with one short circuit. At first sight, knowing three values among these six allows the calculation of the three capacitors $\left(C_{1}, C_{2}\right.$ and $\left.C_{3}\right)$. A deeper examination shows some difficulties in doing this.

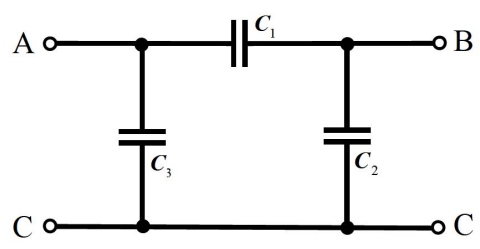

Figure 5. capacitive two-port

To study a numerical example we assign values to capacitors seen in figure 5: $C_{1}=C_{2}=40 \mathrm{pF}$ and $C_{3}=10 \mathrm{pF}$. Now, let us suppose that, on this device, we measure: the input capacitance with the output open $(30 \mathrm{pF})$ and with the output shorted $(50 \mathrm{pF})$ and also the output capacitance with the input shorted $(80 \mathrm{pF})$. Starting from these measurements, we now try to recover the three capacitors values. A simple computation leads to this solution. That's good. Unfortunately, the same computation also gives another solution: $C_{1}=-40 \mathrm{pF}$, $C_{2}=120 \mathrm{pF}$ and $C_{3}=90 \mathrm{pF}$. With this last set of values, all measurable capacitances of the device should be positive so it cannot be eliminated for physical reasons. Nevertheless, some measurable impedances of this circuit differ from the real ones. For example, capacitance seen between $\mathrm{A}$ and $\mathrm{B}$ when $\mathrm{C}$ is floating equals $48 \mathrm{pF}$ for the initial values against $11.43 \mathrm{pF}$ for the second set of values.

In fact, as soon as we measure an impedance which involves two capacitors in series (here the impedance seen between $\mathrm{A}$ and $\mathrm{C}$ with $\mathrm{B}$ floating), we get non linear equations which lead to multiple solutions. Hopefully, if we only acquire measurements in which all the capacitances are in parallel, all corresponding equations are linear so the system has a unique solution. For the device shown in figure 7, measuring the three capacitances seen when a short circuit is present leads only to wanted values.

\section{B. Choice of measurements}

The previous example leads to more general conclusions. In order to identify, with no ambiguity, the electrostatic coupling of an N-ports electrostatic device, measurements must be acquired with a maximum of terminals short-circuited, in order to just see capacitances in parallel. Ideal situation is reached when, during the measurement, terminals are all connected in two separated groups. One can show that there are $2^{N}-1$ such measurements while $N(N+1) / 2$ equations are needed for a complete characterization. Therefore, it is always possible to acquire only measurements of this kind.

In practice, we always keep in mind the previous recommendation. For example, we first characterize the transformer with all its winding shorted. If it owns $\mathrm{n}$ windings, this needs $(n-1) n / 2$ measurements which are chosen among configurations in which all terminals are connected in two groups. Choice of following measurements is guided by considerations exposed in the following section.

\section{PROGRESSIVE REPRESENTATION}

\section{A. Progressive representation}

Previous approach looks fine but, sometimes, it leads to experimental difficulties during the identification. As we say above, capacitances values are deduced from a set of six independent impedance measurements, among which some are acquired with, at least, one winding shorten. Related resonances, which are linked to leakage inductances, are sometimes so high that they are out of the range of the measuring apparatus. In such a case, no capacitance value can be deduced because the number of available independent equations is insufficient.

To overcome this problem, we remarked that, in numerous cases, windings are so strongly coupled that, during common use, all winding voltages are almost proportional. If that was exact, a two winding transformer should have only two independent voltages so its capacitive behavior could be represented by a three capacitor circuit. With this in mind, we choose another voltage base [7]. Among the three independent voltages, we introduced that $\left(V_{2}^{\prime}\right)$ which appears across the leakage inductance (Figure 6). $V_{2}^{\prime}$ is often negligible and corresponding approximation is acceptable as long as no winding is shorted. Mathematically, new voltages $V_{1}, V_{2}^{\prime}$ and $V_{3}^{\prime}$ are linear combinations of previous ones so this operation is a simple base change. Equivalent circuit of figure 6 is obtained, as previously, by linking every couple of its terminal by a capacitor, its terminals being now the 4 points between which the 3 new voltages are defined.

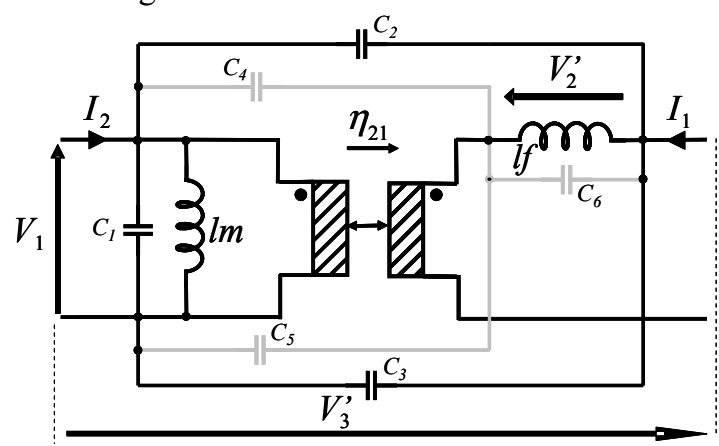

Figure 6. Equivalent circuit for the electrostatic part of a two windings transformer 
This circuit includes six capacitances as the previous one does. Generally speaking, with suitable capacitor values, it is strictly equivalent to circuit of figure 3 . However, if the coupling is strong lf $<<l m$, and as long as lf is negligible (no winding shorted), independent capacitance number reduces to three: $C_{6}$ is shorted, $C_{4}$ and $C_{5}$ are added to respectively $C_{2}$ and $C_{3}$. As a consequence, three independent measures, acquired in these conditions suffice to obtain the three capacitor values. When no winding is shorted, involved inductance is $l m$, which is the stronger one, so needed resonance frequencies are generally accessible to the apparatus.

Owing to this representation, in the worst case, a first approximation which includes three capacitances (placed as those in bold on figure 6) is obtained. Often (but not always), three extra measurements, carried out with one winding shorted, give three extra equations that lead to the values of the six capacitors included in figure 6 .

Extension to $\mathrm{n}$-winding transformers is simple. To draw the equivalent circuit of such a transformer, we first have to locate the $2 \mathrm{n}$ points to which the electrostatic circuit must be connected. The two first ones are the terminals of the winding arbitrarily named primary. Others are connected to all the terminals of the leakage transformer [5]. This transformer represents the leakage inductances and the couplings which do exist between them. Remaining job is simple: we link every couple of terminals by a capacitor.

\section{B. Progressive representation with positive capacitances}

Introducing the capacitance matrix, we only mentioned its symmetry. However, it owns another important property. Because electrostatic energy density is positive, the whole energy stored in the circuit (2) must remain positive whatever the input voltages are. This establishes that capacitance matrix is positive-definite. According to this property, determinants of all its principal sub-matrixes are positive. This causes numerous inequalities among which some are well known: principal values are positives and coupling coefficients are, in absolute values, equal or smaller than one.

Because any measurement includes a part of inaccuracy, all the previous inequality are not surely respected by previous representations (Figures 3 and 6) when their values are deduced from measurements. As a consequence, it is not certain that this equivalent circuit stores a positive energy with all voltage combinations. During a time domain simulation, that can originate negative time constants which prevent the simulation to converge.

An equivalent circuit that represents any linear electrostatic device and that cannot store negative energy is available. This property results directly of the fact that it includes couplers and only positive capacitances. This representation is deduced from our magnetic coupling approach [6], by duality.

Figure 7 includes an equivalent circuit of this kind, with 3 ports, to represent the capacitive behavior of a 2-winding transformer. As former circuits, it is characterized by 6 parameters and, as long as $l f$ is negligible, $C_{3}$ is shorted and so it is for two couplers that, consequently, can be removed. In this situation, the circuit is defined by only 3 parameters. We use this kind of representation to insure electrostatic energy is always positive. This is illustrated below on a practical example.

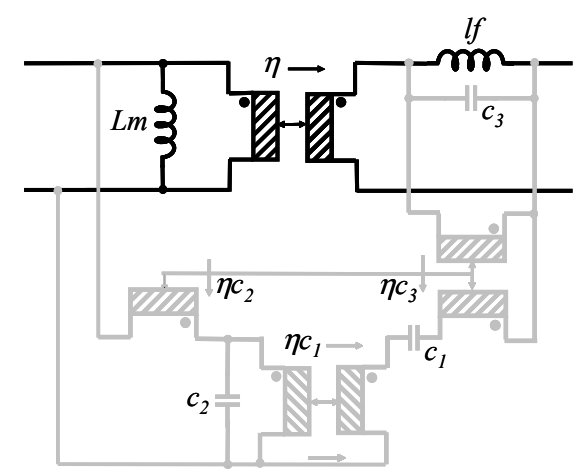

Figure 7. Equivalent circuit with only positive capacitors

\section{EXPERIMENTAL RESULTS}

\section{A. Tested component}

Experimental results presented below are acquired on a 3winding planar transformer built by Thales. It normally works at $125 \mathrm{kHz}$, with power efficiency higher than $98.5 \%$, in a $250 \mathrm{~W}$ power supply. Its magnetic coupling has already been identified (figure 8) according to [6].

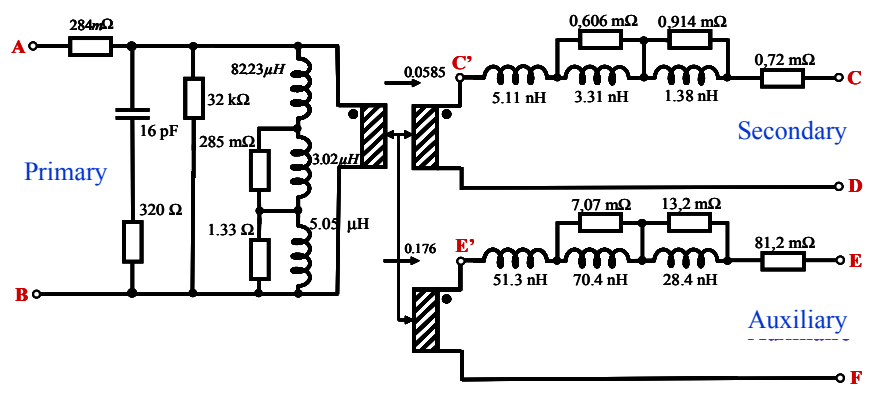

Figure 8. Equivalent circuit of magnetic coupling

While this component work at $125 \mathrm{kHz}$, Bode diagrams of its impedances seem simple (figure 11) up till, at least, $20 \mathrm{MHz}$. For this reason, the first approximation of the progressive representation is, probably, precise enough. So we adopt it as a first step approach. According to this, electrostatic part of the equivalent circuit is a 3-ports capacitive circuit (Figure 9) which is connected to terminals $\mathrm{A}, \mathrm{B}, \mathrm{C}$ and $\mathrm{E}$ (Figure 8 ) of magnetic representation.

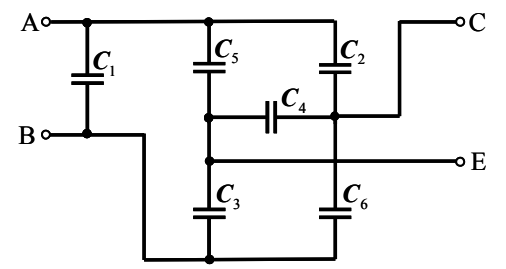

Figure 9. Equivalent circuit of electrostatic coupling

\section{B. Identification with all windings shorted}

To access the values of the six involved capacitors, we first study the behavior with all windings shorted, that is to say 
with A connected to B. The three measurements depicted in table 1 lead to the three capacitances.

TABLE 1. MEASUREMENTS CARRIED OUT WITH ALL WINDINGS SHORTED.

\begin{tabular}{|c|l|}
\hline$m c 1:$ C linked to $\mathrm{E}$ & $\mathrm{Cmc}_{1}=486,5 \mathrm{pF}=\left(C_{3}+C_{5}\right)+\left(C_{2}+C_{6}\right)$ \\
\hline $\begin{array}{c}m c 2: \text { E linked to } \\
\mathrm{A} \& \mathrm{~B}\end{array}$ & $C m c_{2}=498,1 \mathrm{pF}=C_{4}+\left(C_{2}+C_{6}\right)$ \\
\hline $\begin{array}{c}m c 3: \text { C linked to } \\
\mathrm{A} \& \mathrm{~B}\end{array}$ & $C m c_{3}=108,3 \mathrm{pF}=\left(C_{3}+C_{5}\right)+C_{4}$ \\
\hline
\end{tabular}

Knowing these 3 capacitances $C_{2}+C_{6}, C_{3}+C_{5}$ and $C_{4}$, consistency of the representation used for 3 windings shorted can be checked. Capacitance seen between 2 windings when the third is floating have been calculated and measured: differences were smaller than $1 \mathrm{pF}$.

\section{Identification with all windings open}

Figure 9 shows that, in these conditions, 4 distinct wirings are still possible if we gather the four terminals into two groups. They are described in table 2. Joining the three first equations to the three previous ones, we obtain a linear system that gives the six capacitances of the equivalent circuit (Table 3). Unused measure ( $m c 10)$ allows an extra consistency check: the sum of the four capacitances involved gives $444.4 \mathrm{pF}$.

TABLE 2. CAPACITANCES MEASURED BETWEEN A AND B.

\begin{tabular}{|c|l|}
\hline $\begin{array}{c}m c 7: \mathrm{C} \text { and } \mathrm{E} \\
\text { linked to } \mathrm{A}\end{array}$ & $C m_{7}=C_{1}+C_{3}+C_{6}=78,1 \mathrm{pF}$ \\
\hline $\begin{array}{c}m c 8: \mathrm{C} \text { and } \mathrm{E} \\
\text { linked to B }\end{array}$ & $C m c_{8}=C_{1}+C_{2}+C_{5}=376,3 \mathrm{pF}$ \\
\hline $\begin{array}{c}m c 9: \mathrm{C} \text { linked to } \mathrm{A} \\
\text { and E to B }\end{array}$ & $C m c_{9}=C_{1}+C_{4}+C_{5}+C_{6}=133,9 \mathrm{pF}$ \\
\hline $\begin{array}{c}m c 10: \mathrm{C} \text { linked to } \\
\mathrm{B} \text { and E to A }\end{array}$ & $C m c_{10}=C_{1}+C_{2}+C_{3}+C_{4}=444,3 \mathrm{pF}$ \\
\hline
\end{tabular}

TABLE 3. CAPACITANCES OF THE EQUIVALENT CIRCUIT.

\begin{tabular}{|c|l|l|}
\hline$C_{1}=-15.9 p F$ & $C_{2}=370.3 p F$ & $C_{3}=26.2 p F$ \\
\hline$C_{4}=60 p F$ & $C_{5}=22.1 p F$ & $C_{6}=67.9 p F$ \\
\hline
\end{tabular}

We can now compare the curves deduced from our equivalent circuit to those measured. However, it is prudent to check before if electrostatic energy of our circuit remains positive in all circumstances. To know this, we search the parameters of the circuit shown in figure 10 which is equivalent to that of figure 9. Parameter values (Table 4) of the new circuit are found by identifying total energies stored by both circuits.

TABLE 4. PARAMETERS OF SECOND EQUIVALENT CIRCUIT.

\begin{tabular}{|c|c|c|}
\hline$\gamma_{1}=57.1 p F$ & $\gamma_{2}=464.9 p F$ & $\gamma_{3}=108.3 p F$ \\
\hline$\eta_{1}=.823$ & $\eta_{2}=.659$ & $\eta_{3}=.554$ \\
\hline
\end{tabular}

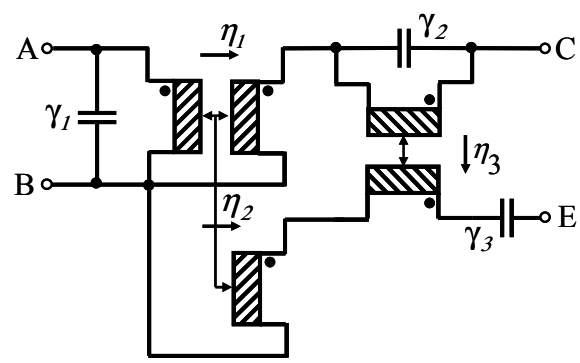

Figure 10. Equivalent circuit of electrostatic coupling including only positive capacitances

That's all right: all capacitances of the second circuit are positive. If it was not the case, we should have verified our measurements and our calculations. At worst, when measurements cannot be very precise, one can join circuit of figure 10 to that of figure 8 and use an optimization algorithm to obtain measured and simulated Bode plots as close as possible while forcing the 3 capacitances to remain positive.

Because our approach is global, our equivalent circuit is able to accounts for all measurable impedances. On a three winding transformer, these impedances are numerous so we present a selection with various kinds of impedances. Other curves and more details about measurements can be found in [8].

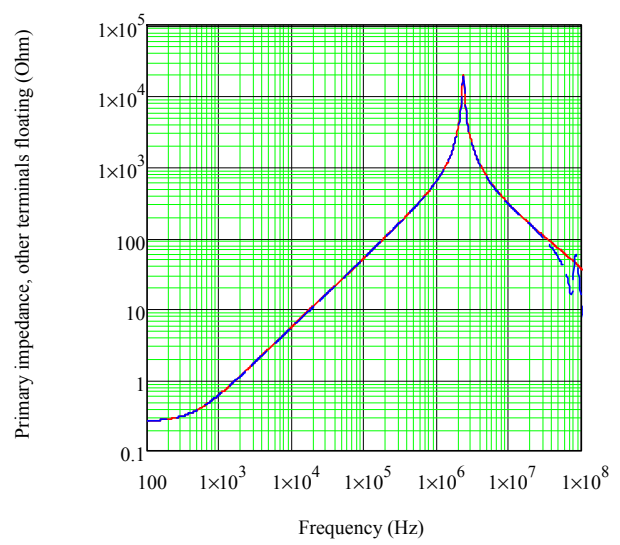

$\boldsymbol{a}$

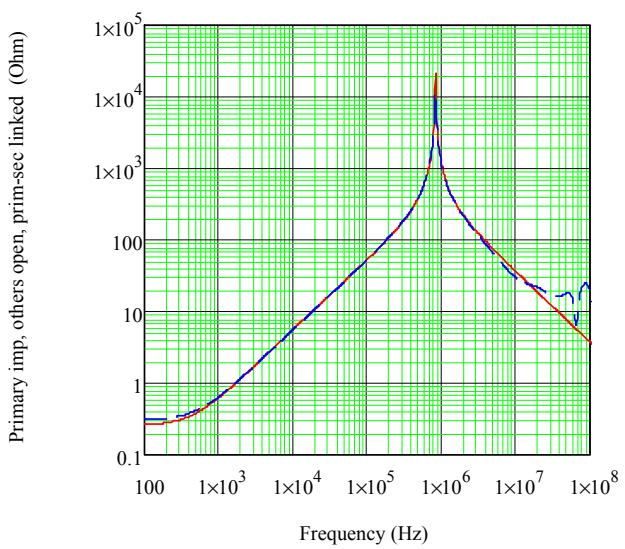

b 


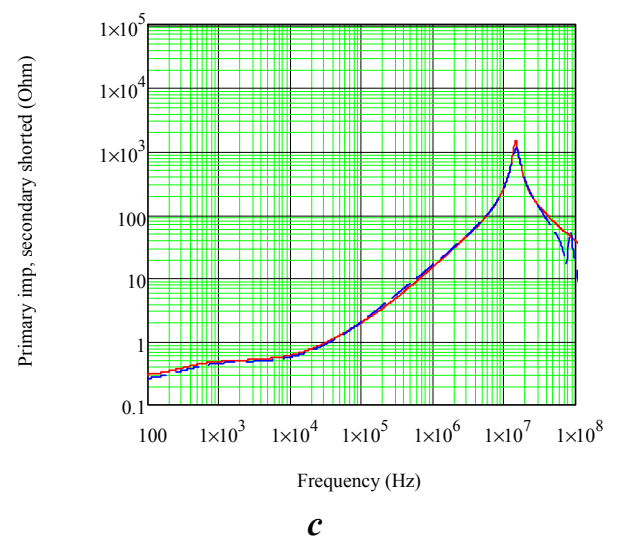

Figure 11. Measurements: blue dashed line, model: red solid line; $\boldsymbol{a}$ ) Primary impedance with other points floating; $\boldsymbol{b}$ ) primay impedance with A linked toC and B linked to E; $c$ ) primary impedance, secondary shorted.

The first set of impedances is seen from the primary side. Our model agrees well with measurement up till about $30 \mathrm{MHz}$. Despite it is perhaps possible to refine this model (high frequency resonances are visible) we stopped here because working frequency is at $125 \mathrm{kHz}$, so the model suits for more than hundred harmonics as it is. Before ending, we found interesting to show the accuracy reached for interwinding impedances which are so important for common mode electromagnetic compatibility.
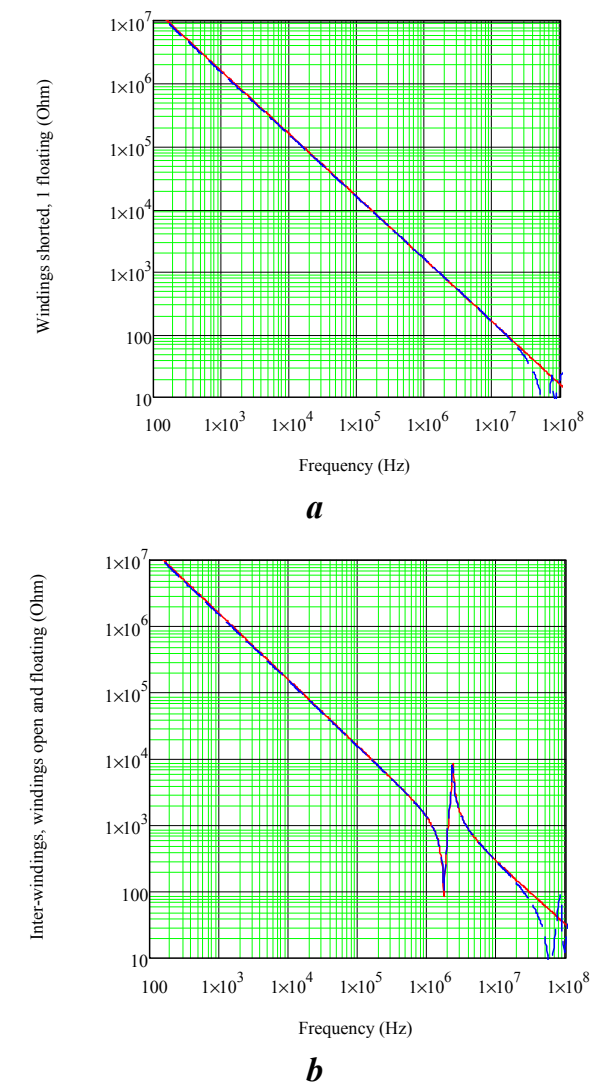

Figure 12. Measurements: blue dashed line, model: red solid line; a) 3 windings shorted but floating; $\boldsymbol{b}$ ) Impedance seen between terminals B and E,

\section{CONCLUSION}

Characterization of the electrostatic behavior of a HF power transformer requires the knowledge of a suitable model. In this paper, we have established such a model, adopting a global approach of the electrostatic behavior. We also showed how wrong values can result from right equations and how to avoid these problems.

Our approach is progressive: it goes through several steps that correspond to more and more precise representations. Obtained model is robust: total electrostatic energy it stores is never negative. This is important to insure time domain simulation convergence. Finally we established the model of an industrial 3-winding transformer and we checked the agreement between its previsions and experimental impedance curves. With only six capacitors, agreement for all impedances is extended from 1 to $30 \mathrm{MHz}$.

Our equivalent circuits are also useful to conclude finite element simulations.

\section{REFERENCES}

[1] J. Biela, J. W. Kolar, "Using transformer parasitics for resonant converters. A review of the calculation of the stray capacitance of transformers", IEEE Transactions on Industry Applications, Jan.-Feb. 2008, vol. 44, no. 1, pp. 223-232.

[2] E. Laveuve, J. P. Keradec, M. Bensoam, "Electrostatic of wound components: analytical results, simulation and experimental validation of the parasitic capacitance", IEEE Industrial Application Society, Dearborn (Mi, USA), 28 Sept. - 4 Oct. 1991, Proc. vol. 2, pp. 1469-1475

[3] F. Blache, J. P. Keradec, B. Cogitore, "Stray capacitances of two winding transformers: equivalent circuit, measurement, calculation and lowering", IEEE Industrial Application Society, Denver (Co, USA), Oct. 2-6 1994, Proc., vol. 2, pp. 1211-1217

[4] B. Cogitore, J. P. Keradec, J. Barbaroux, "Two winding ferrite core transformer: an experimental method to obtain a wide frequency range equivalent circuit", IEEE Transactions on Instrumentation and Measurements, April 1994, vol. 43, no. 2, pp. 364-371.

[5] X. Margueron, J. P. Keradec, "Design of equivalent circuits and characterization strategy for n-input coupled inductors", IEEE Transactions on Industry Applications, Jan.-Feb. 2007, vol. 43, no. 1, pp. 14-22.

[6] X. Margueron, J. P. Keradec, "Identifying the magnetic part of the equivalent circuit of n-winding transformer", IEEE Transactions on Instrumentation and Measurements, Feb. 2007, vol. 56, no. 1, pp. 146-152.

[7] A. Schellmanns, K. Berrouche, J. P. Keradec, "Multiwinding transformers: a successive refinement method to characterize a general equivalent circuit", IEEE Transaction on Instrumentation and Measurements, Oct 1998 , vol. 47, no. 5, pp. 1316-1321.

[8] J. P. Keradec, "Transformateurs HF à $n$ enroulements. Circuits à constantes localisées ", dossier D $3058 \mathrm{Fev}$ 2008 and "Transformateurs HF à $n$ enroulements. Identification expérimentale", dossier D 3 059, Mars 2009, Les Techniques de l'Ingénieur, Paris (F).

http://www.techniques-ingenieur.fr/recherche/ResultList.asp other terminals floating. 